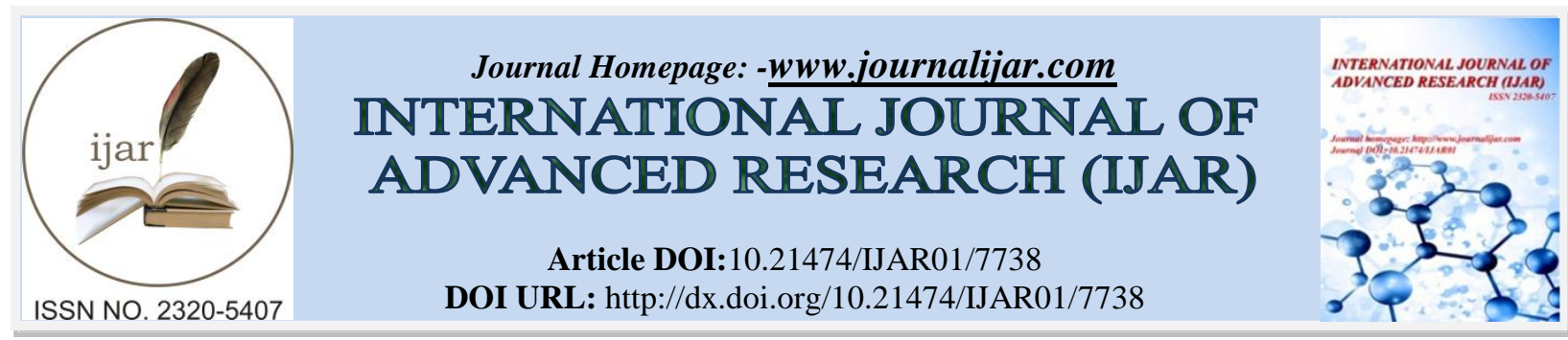

RESEARCH ARTICLE

\title{
PHYTOCHEMICAL ANALYSIS OF LEPIDIUM SATIVUM USING UV-VIS AND GC-MS.
}

\section{Jency Malar .M.S ${ }^{1}$, J. Shifa Vanmathi ${ }^{2}$ and K.Chairman ${ }^{3}$.}

1. Research scholar, Department of Zoology, Manonmaniam Sundaranar University, Abishekapatti.

2. Guest faculty, PG and Research Department of Zoology, St.John's College, Palayamkottai, Tirunelveli.Manonmaniam Sundaranar University, Abishekapatti..

3. Guest faculty, UG and PG Department of Microbiology, Kamarajar Govt. Arts College, Surandai, Manonmaniam Sundaranar University, Abishekapatti.

\section{Manuscript Info}

(..........................

Manuscript History

Received: 23 July 2018

Final Accepted: 29 August 2018

Published: September 2018

Keywords:-

Lepidiumsativum,UV-VIS and GC-

MS,Phytochemical compounds.

\section{Abstract}

The present study was carried out to characterize the bioactive constituents present in seed and whole plant extracts of Lepidium sativum using UV-VIS and GC-MS. The crude extracts were scanned in the wavelength ranging from 200 to $800 \mathrm{~nm}$ by using Perkin Elmer spectrophotometer and the characteristic peaks were detected. For GCMS analysisabout $25 \mathrm{~g}$ of powdered plant material was uniformly packed into a thimble and extracted with $150 \mathrm{ml}$ of ethanol as solvent using this plant extract was prepared. Helium gas (99.999\%) was used as the carrier gas at constant flow rate $1 \mathrm{ml} / \mathrm{min}$ and an injection volume of $2 \mu 1$ was employed (split ratio of $10: 1$ ); Injector temperature $80^{\circ} \mathrm{C}$; Ion-source temperature $250^{\circ} \mathrm{C}$. The oven temperature was programmed from $110^{\circ} \mathrm{C}$ (isothermal for $2 \mathrm{~min}$.), with an increase of $10^{\circ} \mathrm{C} / \mathrm{min}$, to $200^{\circ} \mathrm{C}$, then $5^{\circ} \mathrm{C} / \mathrm{min}$ to $250^{\circ} \mathrm{C}$, ending with a $9 \mathrm{~min}$ isothermal at $280^{\circ} \mathrm{C}$. Mass spectra were taken at $70 \mathrm{eV}$; a scan interval of 0.5 seconds and fragments from 45 to $450 \mathrm{Da}$. The UV-VIS profile showed different peaks ranging from 280 and $290 \mathrm{~nm}$ with absorbance values of 0.26 and 3.98 respectively.The spectra for phenolic compounds (tannins) and flavonoids typically lie in the range of 230-290 $\mathrm{nm}$. The results of the GC-MS analysis provide different peaks determining the presence of 28 phytochemical compounds in seed extract and the major phyto constituents were (Peak area 16.23\%),o-ethyl S-2Dimethylaminoethyl Ethylphos, (14.37\%)Oleoyl chloride and (12.50\%) cis-9-Hexadecenal (8.97\%). Phytochemical compoundspresent in whole plant extract was 79 and the major phyto constituents were Eugenol (7.69 \% ); Hexadecanoic Acid, Ethyl Ester $(7.50 \%)$ and Stigmast-5-EN-3-OL, (3.BETA.)- (7.14 \%) were reported by GC-MS analysis. The results revealed the major compounds are fatty acid esters and alkaloids which showed antioxidant, antimicrobial and anticancer activities. 


\section{Introduction:-}

Herbal medicines are in great demand in both developed and the developing countries in primary healthcare because of their great efficacy and little or no side effects. In India, the indigenous system of medicine namely Ayurvedic, Siddha and Unani have been in existence for several centuries. These traditional systems of medicine together with homoeopathy and folklore medicine continue to play a significant role largely in the health care system of the population (Yadavet al., 2011,). The tribals and rural population of India are highly dependent on medicinal plant therapy for meeting their health care needs. This attracted the attention of several botanist and plant scientists of several medicinal plants and there was a spurt of scientific literature. (Cerutti, 1991).

Plantsare the best sources for chemical ingredients or phytochemical agents for cure of different disease. Medicinal plants are an inexhaustible source of molecules with very different biological and pharmacological activities(KshitijChauhanet al, 2012).Lepidiumsativum Linn (Brassicaceae) commonly known as Asaliyo, is an erect, glabrous annual herb cultivated as a salad plant throughout India, Europe and United States. The seeds are used in chronic enlargement of liver and spleen, as carminative adjunct to purgatives, in skin diseases, dysentery, diarrhoea, asthma and in liver complaints (shuklaet al., 2015).

Lepidiumsativum, Family Brassicaceae, is a fast-growing, edible plant botanically related to watercress and mustard and known to share their peppery, tangy flavour and aroma (Prajapatiet al., 2014). In some regions, garden cress is known as garden pepper cress, pepper grass or pepperwort. Cress is one of the easiest vegetables to grow as it can grow just about anywhere. The plant isused as an antiasthmatic; anti-scorbutic; aperient; diuretic; galactogogue ; poultice and stimulant (Cassidy, 2002).

The total glucosinolates of the seeds of Lepidium sativum revealed the presence of two glucosinolates, glucotropaeolin and gluconasturin. On the other hand, four glucosinolates were isolated from the fresh herb and were identified as 2 - ethyl butyl glucosinolate, methyl glucosinolate (glucocapparin), butylglucosinolate in addition to the glucotropaeolin which was isolated also from the seeds. The glucosinolates were identified by (UV, MS). The individual corresponding isothiocyanates (aglucone) which was obtained by enzymatic hydrolysis of the individual glucosinolates were identified using GC / MS technique (Radwanet al., 2007).

\section{Materials and Methods:-}

\section{Collection of plant materials}

Fresh materials of Lepidium sativum was grown in the laboratory of Sri Paramakalyani College, Alwarkurichi, Tamilnadu, India. The plant was dried in shade and was pulverized using mortor and pestle separately and stored in a closed vessel for further use.

\section{Preparation of plant extracts - Solvent extraction}

Crude plant extract was prepared by Soxhlet extraction method. About $25 \mathrm{~g}$ of powdered plant material was uniformly packed into a thimble and extracted with $150 \mathrm{ml}$ of ethanol as solvent. The process of extraction was continued for $24 \mathrm{htill}$ the solvent in siphon tube of an extractor become colourless. After that the extract was taken in a beaker and kept on hot plate and heated at $30-40^{\circ} \mathrm{C}$ till the solvent got evaporated. Dried extract was kept in refrigerator at $4^{\circ} \mathrm{C}$ for their future use in phytochemical analysis (Martins et al., 2001).

\section{Ultraviolet-visible spectroscopy analysis:}

The extracts of L.sativum were examined under visible and UV light for proximate analysis. For UV-VIS spectrophotometer analysis, the extracts were centrifuged at $3000 \mathrm{rpm}$ for $10 \mathrm{~min}$ and filtered through Whatmann No. 1filter paper by using high pressure vacuum pump. The sample is diluted to 1:10 with the ethanol. The extracts were scanned in the wavelength ranging from 200-800 nm using Spectrophotometer and the characteristic peaks were detected. The peak values of the UV-VIS were recorded. Each and every analysis was repeated twice for the spectrum confirmation (AH and Aysel 2003).

\section{Gas Chromatography Mass Spectrum (GC-MS) Analysis:}

GC-MS analysis of the extract was performed using a Thermo GC -Trace ultra Ver: 5.0 system and Gas chromatograph interfaced to a Mass spectrometer (GC-MS)(Perkin-Elmer GC Clarus 500 system) equipped with TR 5 - MS capillary standard non-polar column (30mmX0.25mm 1D X $1 \mu \mathrm{Mdf})$. For GC-MS detection, an electron ionization system with ionizing energy of $70 \mathrm{eV}$ was used. Helium gas $(99.999 \%)$ was used as the carrier gas at 
constant flow rate $1 \mathrm{ml} / \mathrm{min}$ and an injection volume of $2 \mu \mathrm{l}$ was employed (split ratio of 10:1); Injector temperature $80^{\circ} \mathrm{C}$; Ion-source temperature $250^{\circ} \mathrm{C}$. The oven temperature was programmed from $110^{\circ} \mathrm{C}$ (isothermal for $2 \mathrm{~min}$.), with an increase of $10^{\circ} \mathrm{C} / \mathrm{min}$, to $200^{\circ} \mathrm{C}$, then $5^{\circ} \mathrm{C} / \mathrm{min}$ to $250^{\circ} \mathrm{C}$, ending with a 9 min isothermal at $280^{\circ} \mathrm{C}$. Mass spectra were taken at $70 \mathrm{eV}$; a scan interval of 0.5 seconds and fragments from 45 to 450 Da. Relative quantities of the chemical compounds present in each of the extracts of L. sativumwas expressed as percentage based on peak area produced in the chromatogram. ( Merlin et al., 2001).

\section{Results and Discussion:- \\ Uv-visible spectrographic analysis:}

The UV-VIS profile of the plant extract was studied at a wavelength range of 200 to $800 \mathrm{~nm}$. Two major peaks were recorded at 280 and $290 \mathrm{~nm}$ with absorbance values of 0.26 and 3.98 respectively. (Table 2.4 and Figure 2.2 ) The spectra for phenolic compounds (tannins) and flavonoids typically lie in the range of 230-290 nm. The result of UVVIS spectroscopic analysis confirms the presence of tannins and flavonoids in the ethanolic extract of L.sativum.

The phenolic contents of methanolic extract of Lepidium sativum was determined by UV spectrophotometric method. The total contentof phenolic compounds were found to be $46.0 \mathrm{mg}$ GAE/100 g in methanolic extract of Lepidium sativum. The flavonoids content was determined by UV spectrophotometric method. The total content of flavonoids was found to be $4.28 \mathrm{mg} \mathrm{QE} / 100 \mathrm{~g}$ in methanolic extract of Lepidium sativumrespectively (RizwanAhamadet al., 2015).

\section{Gas Chromatography Mass Spectrum (GC-MS) Analysis:}

Bioactives are chemical compounds often referred to as secondary metabolites. The identification of bioactive chemical compounds is based on the peak are, retention time molecular weight and molecular formula are presented in table 2.5 and figure 2.3 and 2.5.1. Eleven major bioactive compounds were identified in the ethanol seed extract of Lepidium sativum. GC-MS analysis of Lepidium sativum seed extracts revealed the existence of Benzyl nitrile (Peak area 16.23\%),o-ethyl S-2-DIMETHYLAMINOETHYL ETHYLPHOS (14.37\%)Oleoyl chloride (12.50\%) cis9-Hexadecenal (8.97\%) 3',5'-Dimethoxyacetophenone (7.93\%) gamma.-Sitosterol (7.39\%) ETHYL (9Z,12Z)9,12-OCTADECADIENOATE (4.96\%) n-Hexadecanoic acid ( $4.19 \%)$ gamma.-Tocopherol (4.01\%) Benzene, (isothiocyanatomethyl)- (3.89 \%)and ERGOST-5-EN-3-OL, (3.BETA.,24R)- ( 2.23\%) and also the minor compounds were HEXADECANOIC ACID, ETHYL ESTER (1.87\%), Fumaric acid, 2-dimethylaminoethyl nonyl ester (1.30\%), Hexadecanoic acid , 2-hydroxyl-1-(hydroxymethyl) ethyl ester (1.14\%) and STIGMASTA -5-24(28)DIEN-3-OL,(3.BETA)- respectively.

The bioactive compounds in ethanol extract of Lepidium sativium of whole plant by GC-MS report was reported in Table 2.6 and Figure 2.4 and 2.6.1. The chromatogram of GC-MS analysis showed the presence of 26 major constituents are m Eugenol (7.69 \% ); HEXADECANOIC ACID, ETHYL ESTER (7.50\%); STIGMAST-5-EN-3OL, (3.BETA.)- (7.14 \%),Dichloroacetic acid, tridec-2-ynyl ester (6.70\% ). Stigmastane-3,6-dione, (5.alpha.)- (5.05 $\%$ ) 9,12-Octadecadienoyl chloride, (4.51\% ); gamma.-Sitostenone (4.10\%) ,Cholestan-3-one, 4,4-dimethyl-, (5.alpha.) (3.58\%)-, n-Hexadecanoic acid (3.15\%); Stigmasterol (2.86\%); trans,trans-9,12-Octadecadienoic acid, propyl ester (2.54\%); ERGOST-5-EN-3-OL, (3.BETA.,24R)- 2.38\%) :CYCLODODECANONE,2-(HYDROXY BUTYL)-2-NIT (2.35\%) and along with other minor constituents were Benzyl nitrite (1.32\%), Benzoic acid (1.47\%), Dodecanoic acid $(1.01 \%)$, (E)-9-Octadecenoic acid ethyl ester (1.98\%), OCTADECANOIC ACID, ETHYL ESTER (1.50\%), (E)-9-Octadecenoic acid ethyl ester( 1.67\%), Behenic alcohol (1.35\%), Hexacosylheptafluoro butyrate(1.52\%), Ethyl tetra cosanoate (1.52\%), Cyclohexyldimethylsilyloxy butane, 1-Heptacosanol, Cholesterol, 4Campestene-3-one, 4,22-Stigmastadiene-3-one (1.40\%) were reported by GC-S analysis. The results revealed the major compounds are fatty acid esters and alkaloids which showed antioxidant, antimicrobial and anticancer activities.

Lepidium sativum contained several flavonoids, including two quercetin-hexosidesthat shared $[\mathrm{M}-\mathrm{H}]-$ at $\mathrm{m} / \mathrm{z} 463$, identified through the loss of sugar moieties (probably glucose and galactose units) and resultant ionization of quercetin at $\mathrm{m} / \mathrm{z} 301$. More phenolics have been identified in L. sativum. The chromatographic profile of some polyphenols identified in L. sativum is reported. At least three isomers of caffeoylquinic acid were identified in the seeds of L. sativum(Muhammad Zia-Ul-Haqet al., 2012).

The GC-MS result of the L. sativum oil revealed that total 17 fatty acid methyl esters were determined in the esterified oil. The major component of L. sativiumseed oil was docosatrienoic acid (C22:3; 47.66\%) followed by linoleic acid (C18:2; 11.51\%), eicosenoic acid (C20:1; 10.63\%), palmitic acid (C16:0; 10.13\%), arachidonoic acid 
(C20:4; 4.70\%), eruic acid (C22:1; 4.40\%), stearic acid (C18:0; 3.34\%), and arachidic acid (C20:0; 3.23\%). L. sativium seed oil was composed of total mono-unsaturated fatty acids (16.32\%), poly-unsaturated fatty acids $(65.35 \%)$, and saturated fatty acid $(18.31 \%)$. This study was supported by Al-Jasass and Al-Jasser (2012) research works in which the percentages of total saturated and unsaturated fatty acids was reported as $16.76 \%$ and $83.24 \%$, respectively (Solomon et al., 2015).

GC-MS analysis determined the 15 compounds in total alkaloid extract of Lepidium sativum seeds. The compounds are methyl (Z)-5,11,14,17-eicosatetraenoate (10.24\%), guanosine (9.29\%), dodecanamide, n-(2-hydroxyethyl) (7.48\%), hexadecanoic acid, 2-hydroxy-1-(hydroxymethyl) ethyl ester (7.13\%), 1-(1-adamantyl)-3-(1-piperidinyl)1-propanone (6\%), hexadecanoic acid (5.33\%), 3-butylindolizidine (4.80\%), 9,12-octadecadienoic acid (Z,Z)-, 2hydroxy-1-hydroxymethyl (4.79\%), 3- methyl alpha.-d-glucopyranoside (1.81\%), stigmast-5-en-3-ol, (3.beta.) $(3.58 \%)$, soyasapogenol B (1.15\%), stigmasterol (1.07\%), fucosterol (3.29\%), gamma.-tocopherol (5.04\%) and squalene (3.44\%). The results revealed the major compounds are fatty acid esters and alkaloids which showed antioxidant, antimicrobial, anticancer, antineuropathic, anti-inflammatory activities (Akashet al., 2017). The most effective ingredient present in LS is isothiocyanates, which is formed with glucosinolates (Kassieet al., 2002).

The Chromatogram GC-MS analysis of the methanol extract of Lepidium sativumshowed the presence of thirty one major peaks and the components corresponding to the peaks were determined to be Glycerin, Monoethanolamine , 1-Deoxy-d-mannitol , 1-Nitro-2-propanol , 2-Butanamine , (S)- , Furfural , Allylisothiocyanate , Paromomycin, 2Hydroxy-2-(5-methylfuran-2-yl)1-phenylethanone, 3,6-Diazahomoadamantan-9-one Hydrazone, 2,3,4Trimethoxycinnamic acid, 2-Naphthalenol, 2,3,4,4a,5,6,7-octahydro-1,4a-dimethyl-7-(2)-, cis-Vaccenic acid, 9Octadecenamide , $\gamma$-Tocopherol , Phthalic acid, decyl oct-3-yl ester, Ergosta-5,22-dien-3-ol,acetate , ( 3及,22E)- , Campesterol and Cholest-5-en-3-ol ,24-propylidene-,(3ß).(Hussein et al., 2017).

In recent years GC-MS studies have been increasingly applied for the analysis of medicinal plants as this technique has proved to be a valuable method for the analysis of non-polar components and volatile essential oil, fatty acids, lipids and alkaloids.

The GC-MS chromatogram shows the peak area separation of the components. The above mentioned isolated compounds from the ethanol extract of Lepidium sativium seem to possess the reported biological activity and further study of these phytoconstituents may prove the medicinal importance in future.

Fig. 2.2:- Uv-vis spectra of ethanolic extract of Lepidium sativum

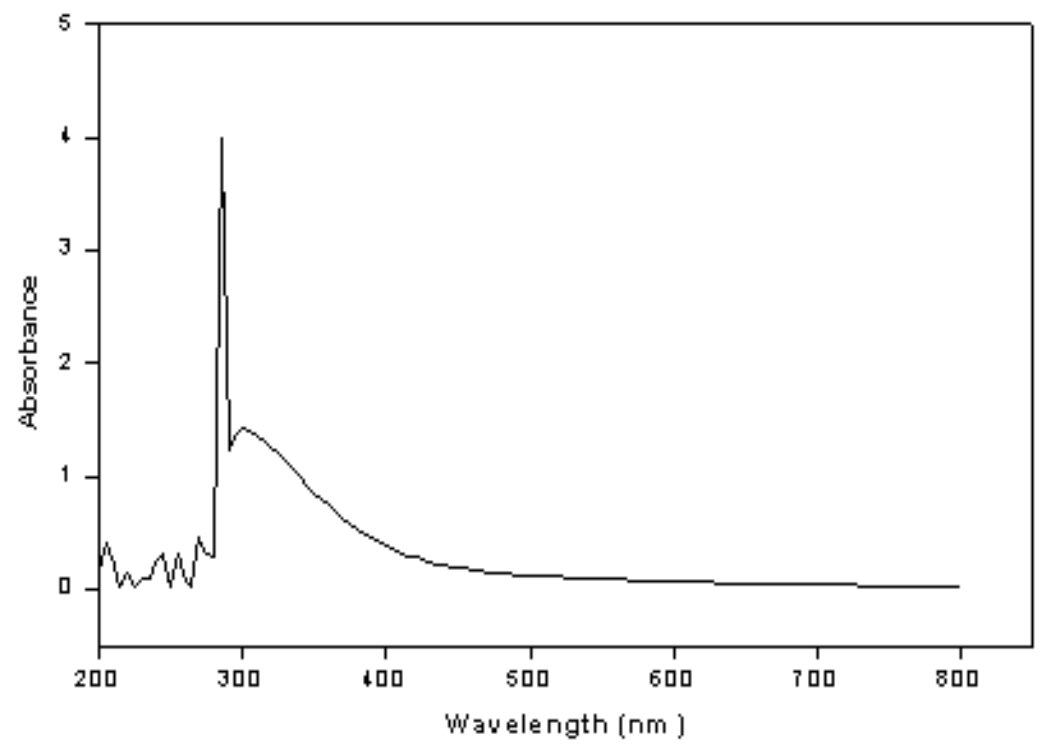


Fig. 2.3:-GC-MS Profile of Ethanol seed extract of Lepidium sativum

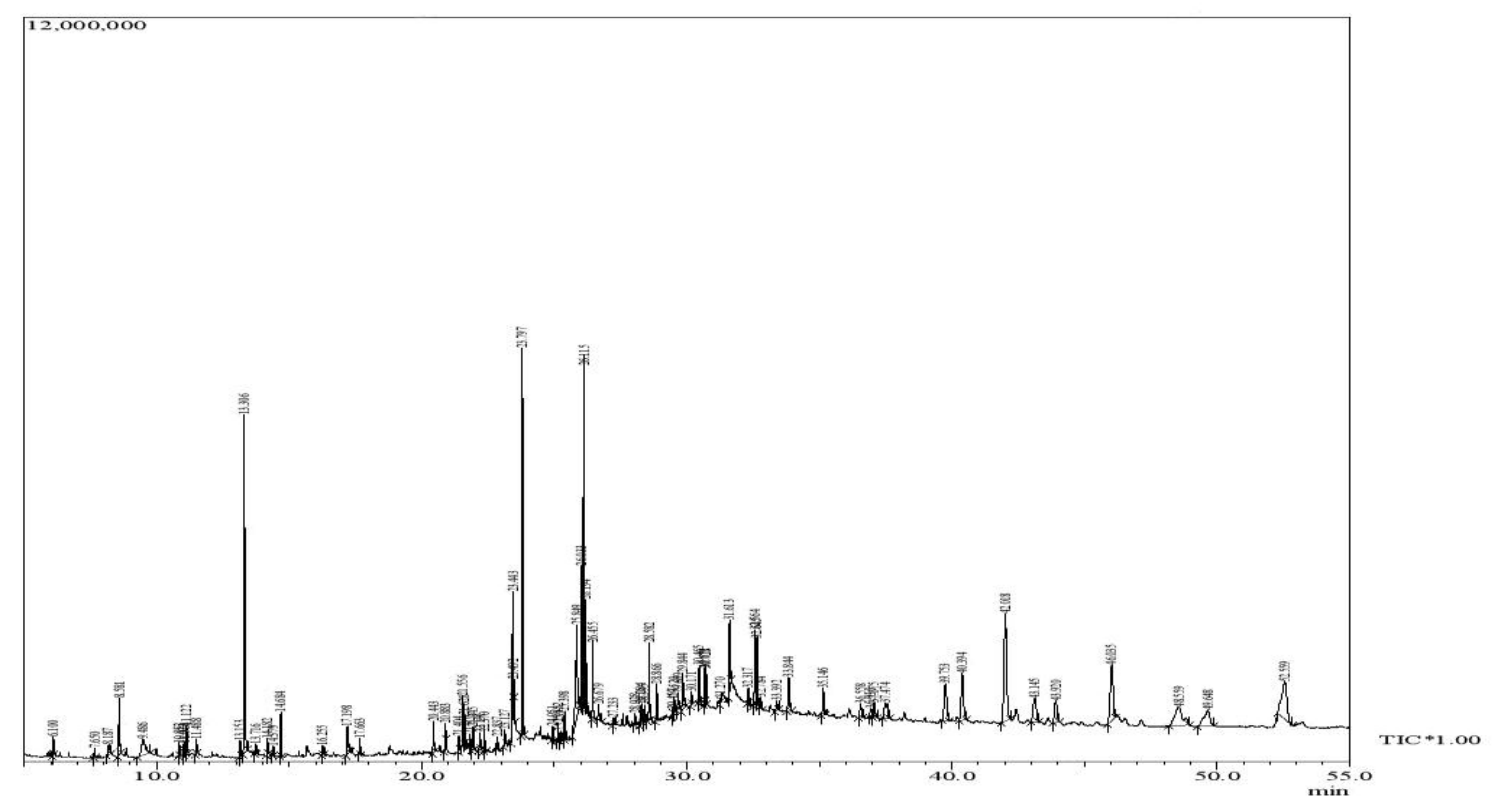

Fig. 2.4:-GC-MS Profile of Ethanol whole plant extract of Lepidium sativum

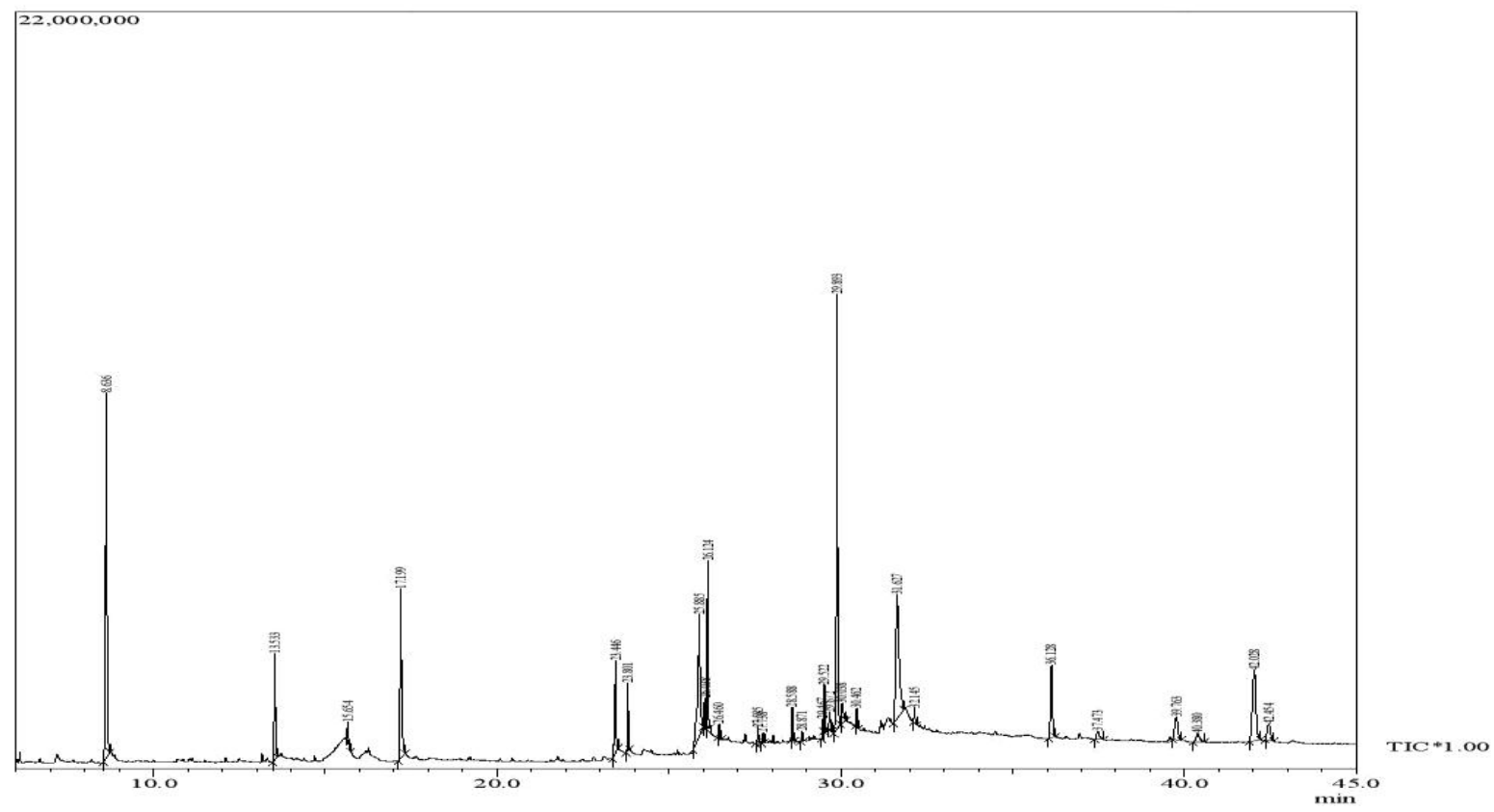

Table: 2.5:-Phytocomponents identified in the ethanol seed extracts of Lepidiumsativum by GCMS analysis.

\begin{tabular}{|l|l|l|l|l|l|}
\hline SI.No & Name of compounds & $\begin{array}{l}\text { Retention } \\
\text { time(min) }\end{array}$ & $\begin{array}{l}\text { Molecular } \\
\text { formula }\end{array}$ & $\begin{array}{l}\text { Molecular } \\
\text { weight }\end{array}$ & $\begin{array}{l}\text { Peak } \\
\text { area \% }\end{array}$ \\
\hline $\mathbf{1 .}$ & Benzyl nitrile & $\mathbf{8 . 6 3 6}$ & $\mathbf{C}_{\mathbf{8}} \mathbf{H}_{7} \mathbf{N}$ & $\mathbf{1 1 7}$ & $\mathbf{1 6 . 2 3}$ \\
\hline $\mathbf{2 .}$ & Benzene, (isothiocyanatomethyl)- & $\mathbf{1 3 . 5 3 3}$ & $\mathbf{C}_{\mathbf{8}} \mathbf{H}_{7} \mathbf{N S}$ & $\mathbf{1 4 9}$ & $\mathbf{3 . 8 9}$ \\
\hline 3. & Benzoic acid, 2-(dimethylamino)ethyl ester & 15.654 & $\mathrm{C}_{11} \mathrm{H}_{15} \mathrm{NO}_{2}$ & 193 & 0.67 \\
\hline $\mathbf{4 .}$ & $\mathbf{3}, \mathbf{5}$ '-Dimethoxyacetophenone & $\mathbf{1 7 . 1 9 9}$ & $\mathbf{C}_{\mathbf{1 0}} \mathbf{H}_{\mathbf{1 2}} \mathbf{O}_{\mathbf{3}}$ & $\mathbf{1 8 0}$ & $\mathbf{7 . 9 3}$ \\
\hline $\mathbf{5 .}$ & n-Hexadecanoic acid & $\mathbf{2 3 . 4 4 6}$ & $\mathbf{C}_{\mathbf{1 6}} \mathbf{H}_{\mathbf{3 2}} \mathbf{O}_{\mathbf{2}}$ & $\mathbf{2 5 6}$ & $\mathbf{4 . 1 9}$ \\
\hline 6. & HEXADECANOIC ACID, ETHYL ESTER & 23.801 & $\mathrm{C}_{18} \mathrm{H}_{36} \mathrm{O}_{2}$ & 284 & 1.87 \\
\hline $\mathbf{7 .}$ & cis-9-Hexadecenal & $\mathbf{2 5 . 8 8 5}$ & $\mathbf{C}_{\mathbf{1 6}} \mathbf{H}_{\mathbf{3 0}} \mathbf{O}$ & $\mathbf{2 3 8}$ & $\mathbf{8 . 9 7}$ \\
\hline
\end{tabular}




\begin{tabular}{|c|c|c|c|c|c|}
\hline 8. & $\begin{array}{ll}\text { ETHYL } & (9 Z, 12 Z)-9,12- \\
\text { OCTADECADIENOATE \# } & \end{array}$ & 26.038 & $\mathrm{C}_{20} \mathrm{H}_{36} \mathrm{O}_{2}$ & 308 & 0.83 \\
\hline 9. & $\begin{array}{ll}\text { ETHYL } & (9 \mathrm{Z}, 12 Z)-9,12- \\
\text { OCTADECADIENOATE \# } & \end{array}$ & 26.124 & $\mathrm{C}_{20} \mathrm{H}_{36} \mathrm{O}_{2}$ & 308 & 4.96 \\
\hline 10. & Octadecanoic acid, ethyl ester & 26.460 & $\mathrm{C}_{20} \mathrm{H}_{40} \mathrm{O}_{2}$ & 312 & 0.40 \\
\hline 11. & 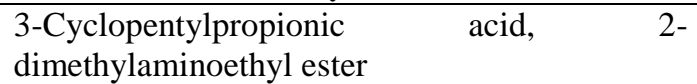 & 27.585 & $\mathrm{C}_{12} \mathrm{H}_{23} \mathrm{NO}_{2}$ & 213 & 0.44 \\
\hline 12. & Glycidylpalmitate & 27.738 & $\mathrm{C}_{19} \mathrm{H}_{36} \mathrm{O}_{3}$ & 312 & 0.30 \\
\hline 13. & (E)-9-Octadecenoic acid ethyl ester & 28.588 & $\mathrm{C}_{18} \mathrm{H}_{34} \mathrm{O}_{2}$ & 282 & 0.95 \\
\hline 14. & Eicosanoic acid, ethyl ester & 28.871 & $\mathrm{C}_{22} \mathrm{H}_{44} \mathrm{O}_{2}$ & 340 & 0.26 \\
\hline 15. & $\begin{array}{l}\text { 3-Cyclopentylpropionic } \\
\text { dimethylaminoethyl ester }\end{array} \quad$ acid, & 29.467 & $\mathrm{C}_{12} \mathrm{H}_{23} \mathrm{NO}_{2}$ & 213 & 0.36 \\
\hline 16. & Fumaric acid, 2-dimethylaminoethyl nonyl ester & 29.522 & $\mathrm{C}_{17} \mathrm{H}_{31} \mathrm{NO}_{4}$ & 313 & 1.30 \\
\hline 17. & Glycidyloleate & 29.672 & $\mathrm{C}_{18} \mathrm{H}_{31} \mathrm{C}_{1} \mathrm{O}$ & 298 & 0.70 \\
\hline 18. & $\begin{array}{l}\text { O-ETHYL S-2-DIMETHYLAMINOETHYL } \\
\text { ETHYLPHOS }\end{array}$ & 29.893 & $\mathrm{C}_{8} \mathrm{H}_{20} \mathrm{NO}_{2} \mathrm{PS}$ & 225 & 14.37 \\
\hline 19. & $\begin{array}{l}\text { Hexadecanoic acid, } \\
\text { (hydroxymethyl)ethyl ester }\end{array}$ & 30.038 & $\mathrm{C}_{19} \mathrm{H}_{38} \mathrm{O}_{4}$ & 330 & 1.14 \\
\hline 20. & Erucic acid & 30.462 & $\mathrm{C}_{22} \mathrm{H}_{42} \mathrm{O}_{2}$ & 338 & 0.55 \\
\hline 21. & Oleoyl chloride & 31.627 & $\mathrm{C}_{18} \mathrm{H}_{33} \mathrm{C}_{1} \mathrm{O}$ & 300 & 12.50 \\
\hline 22. & (Z)-18-Octadec-9-enolide & 32.145 & $\mathrm{C}_{18} \mathrm{H}_{32} \mathrm{O}_{2}$ & 280 & 0.46 \\
\hline 23. & .gamma.-Tocopherol & 36.128 & $\mathrm{C}_{28} \mathrm{H}_{48} \mathrm{O}_{2}$ & 416 & 4.01 \\
\hline 24. & Cholesterol & 37.473 & $\mathrm{C}_{27} \mathrm{H}_{46} \mathrm{O}$ & 386 & 0.67 \\
\hline 25. & ERGOST-5-EN-3-OL, (3.BETA.,24R)- & 39.763 & $\mathrm{C}_{28} \mathrm{H}_{48} \mathrm{O}$ & 400 & 2.23 \\
\hline 26. & Stigmasterol & 40.380 & $\mathrm{C}_{29} \mathrm{H}_{48} \mathrm{O}$ & 412 & 0.85 \\
\hline 27. & .gamma.-Sitosterol & 42.028 & $\mathrm{C}_{29} \mathrm{H}_{50} \mathrm{O}$ & 414 & 7.39 \\
\hline 28. & $\begin{array}{l}\text { STIGMASTA-5,24(28)-DIEN-3-OL, } \\
\text { (3.BETA.)- }\end{array}$ & 42.454 & $\mathrm{C}_{29} \mathrm{H}_{48} \mathrm{O}$ & 412 & 1.59 \\
\hline
\end{tabular}

Structure Of Compounds Isolated In Lepidium Sativum Seed Extract

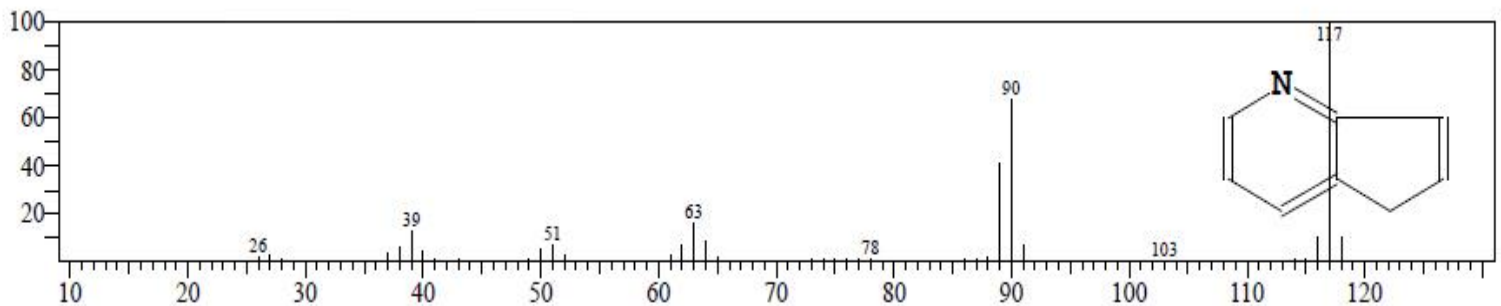

Fig 2.5.1:-Mass Spectrum of Benzyl nitrile

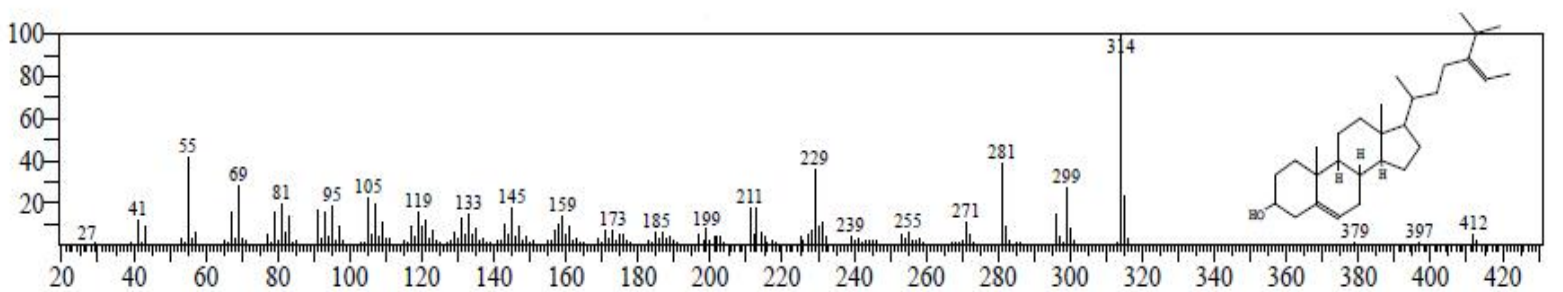

Fig 2.5.2:- Mass Spectrum of Benzene, (isothiocyanatomethyl)- 


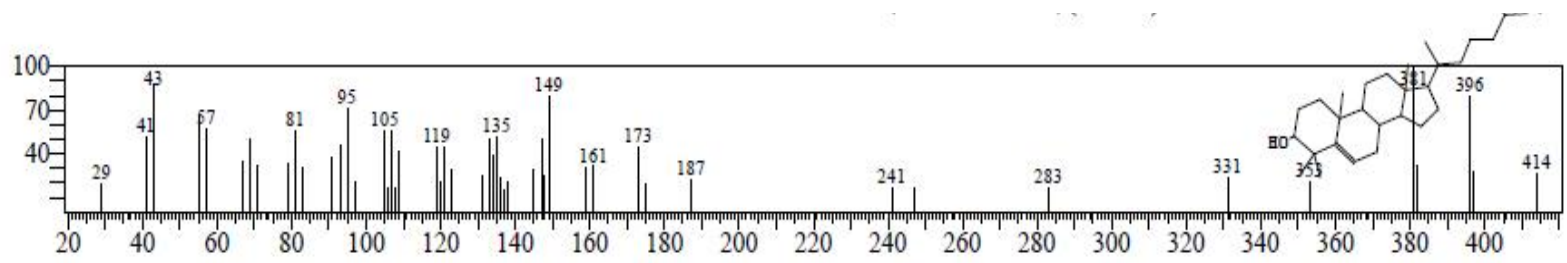

Fig 2.5.3:- Mass Spectrum of 3',5'-Dimethoxyacetophenone

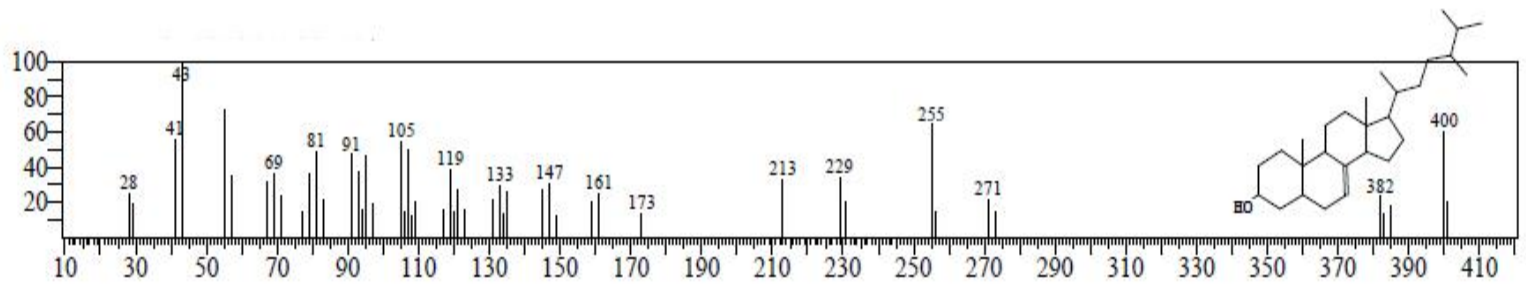

Fig 2.5.4:- Mass Spectrum of n-Hexadecanoic acid

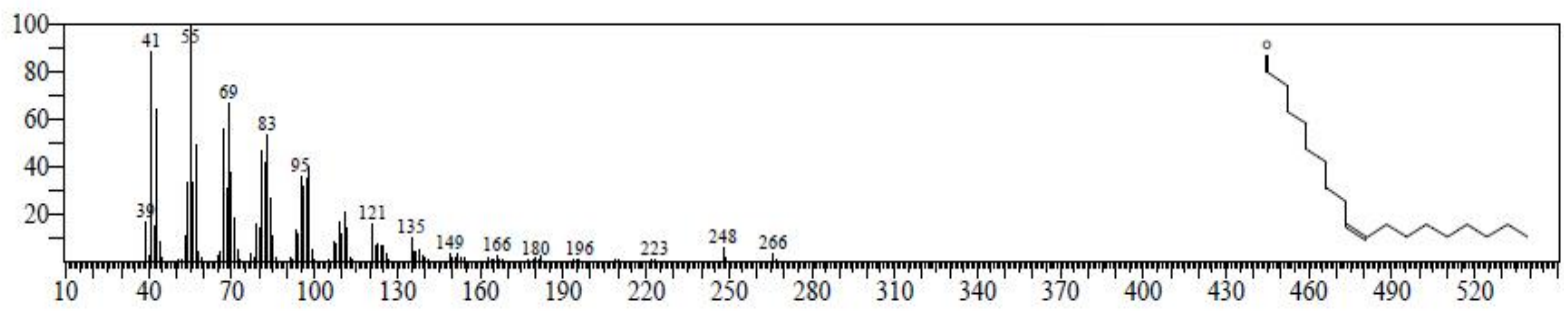

Fig 2.5.6:- Mass Spectrum of cis-9-Hexadecenal

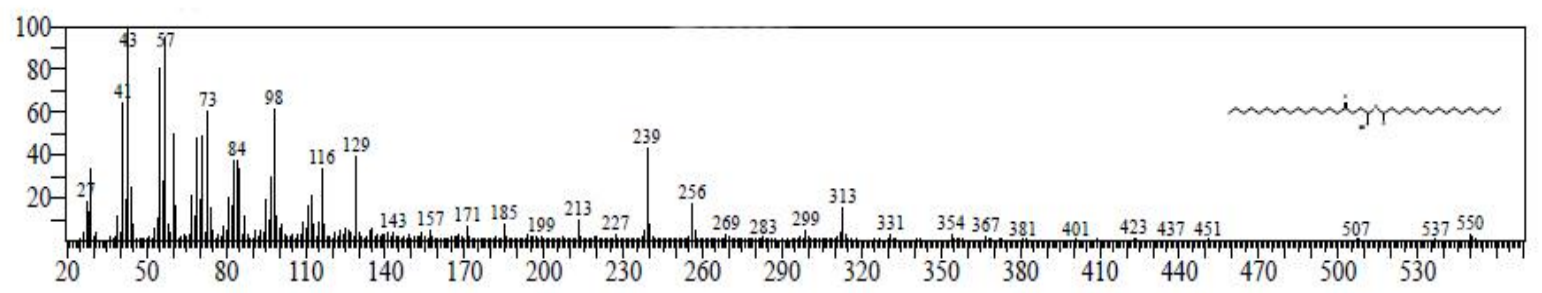

Fig 2.5.7:- Mass Spectrum of Ethyl (9Z,12Z)-9,12-Octadecadienoate \#

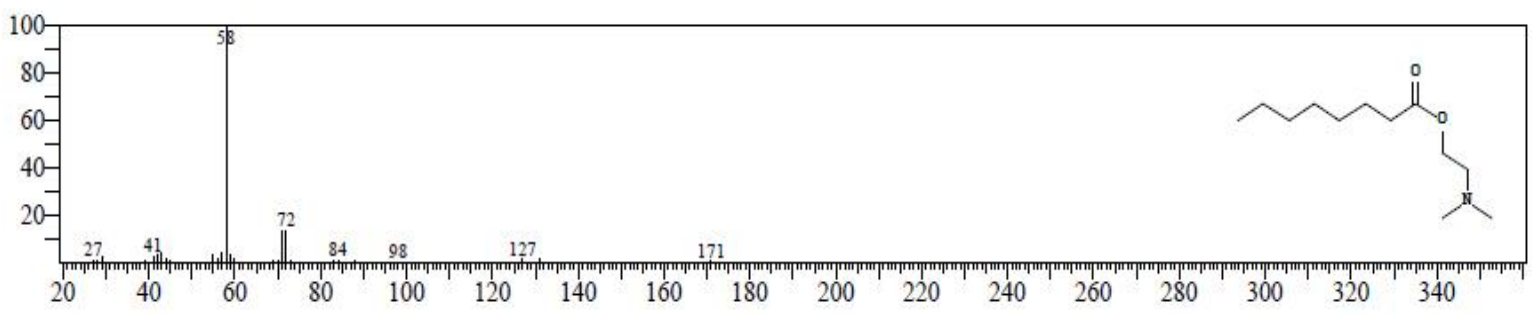

Fig 2.5.8:- Mass Spectrum of O-Ethyl S-2-Dimethylaminoethyl Ethylphos 


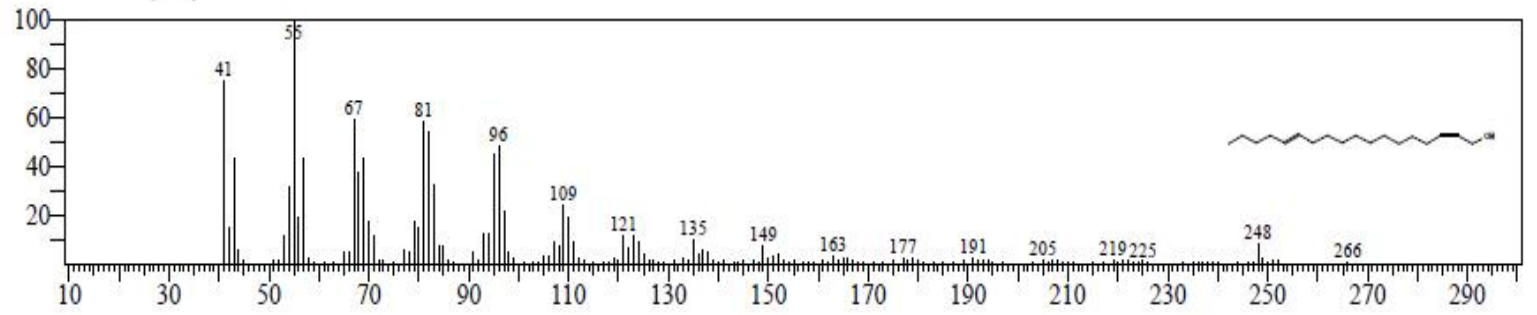

Fig 2.5.9:- Mass Spectrum of Oleoyl chloride

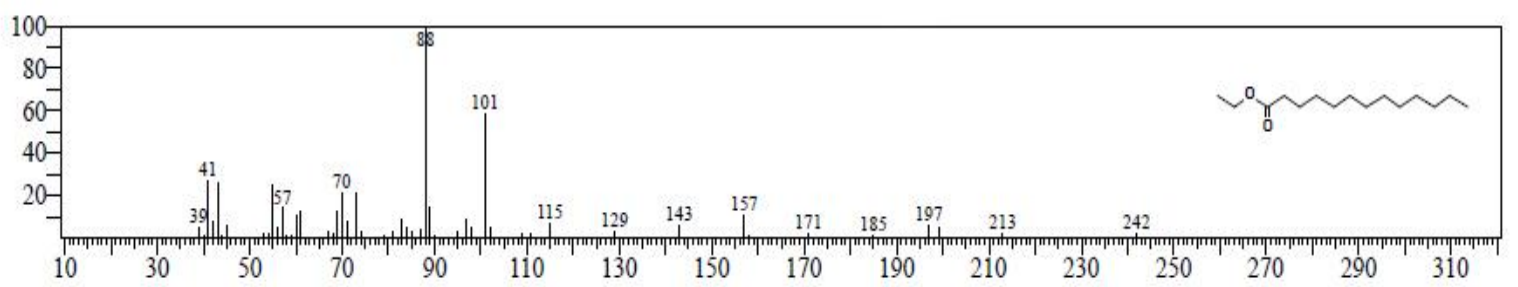

Fig 2.5.10:- Mass Spectrum of.gamma.-Tocopherol

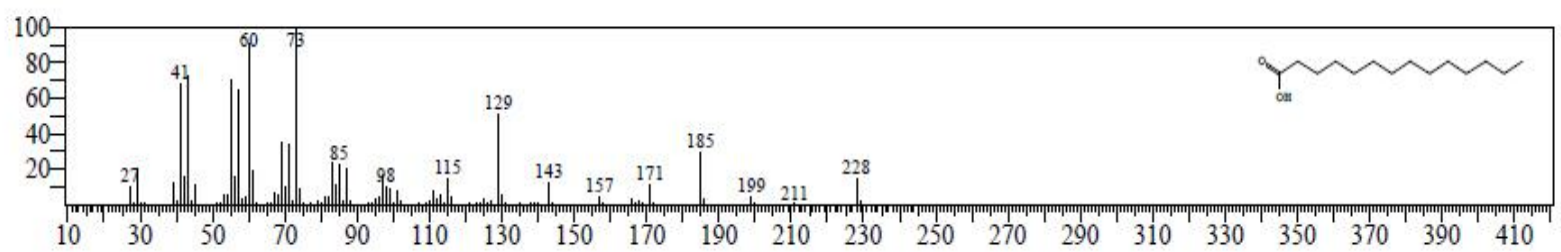

Fig 2.5.11:- Mass Spectrum of Ergost-5-EN-3-OL, (3.Beta.,24R)-

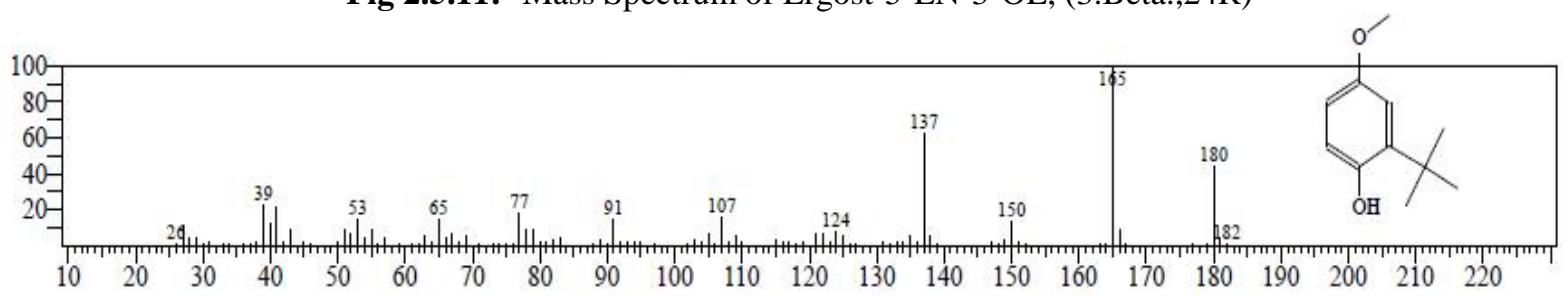

Fig 2.5.12:- Mass Spectrum of.gamma.-Sitosterol

Table2.6.:-Phytocomponents identified in the ethanol whole plant extracts of Lepidiumsativumusing GC-MS analysis

\begin{tabular}{|c|l|l|l|l|l|}
\hline Sl.No & Name of compounds & $\begin{array}{l}\text { Retention } \\
\text { time (min) }\end{array}$ & $\begin{array}{l}\text { Molecular } \\
\text { formula }\end{array}$ & $\begin{array}{l}\text { Molecular } \\
\text { weight }\end{array}$ & $\begin{array}{l}\text { Peak } \\
\text { area \% }\end{array}$ \\
\hline 1. & Eucalyptol & 6.100 & $\mathrm{C}_{10} \mathrm{H}_{18} \mathrm{O}$ & 154 & 0.32 \\
\hline 2. & Linalool & 7.650 & $\mathrm{C}_{10} \mathrm{H}_{18} \mathrm{O}$ & 154 & 0.17 \\
\hline 3. & Benzyl isocyanate & 8.187 & $\mathrm{C}_{8} \mathrm{H}_{7} \mathrm{NO}$ & 133 & 0.36 \\
\hline 4. & Benzyl nitrile & 8.581 & $\mathrm{C}_{8} \mathrm{H}_{7} \mathrm{~N}$ & 117 & 1.32 \\
\hline 5. & Benzoic acid & 9.486 & $\mathrm{C}_{7} \mathrm{H}_{6} \mathrm{O}_{2}$ & 122 & 1.47 \\
\hline 6. & 2,6-Octadienal, 3,7-dimethyl-, (Z)- & 10.852 & $\mathrm{C}_{10} \mathrm{H}_{16} \mathrm{O}$ & 152 & 0.23 \\
\hline 7. & Carvone & 11.021 & $\mathrm{C}_{10} \mathrm{H}_{14} \mathrm{O}$ & 150 & 0.12 \\
\hline 8. & Linalyl acetate & 11.063 & $\mathrm{C}_{12} \mathrm{H}_{20} \mathrm{O}_{2}$ & 196 & 0.11 \\
\hline 9. & Geraniol & 11.122 & $\mathrm{C}_{10} \mathrm{H}_{18} \mathrm{O}$ & 154 & 0.54 \\
\hline 10. & Citral & 11.488 & $\mathrm{C}_{10} \mathrm{H}_{16} \mathrm{O}$ & 152 & 0.28 \\
\hline 11. & $\begin{array}{l}\text { 3-CYCLOHEXENE-1-METHANOL, } \\
\text {.ALPHA.,.ALPHA.,4- }\end{array}$ & 13.153 & $\mathrm{C}_{12} \mathrm{H}_{20} \mathrm{O}_{2}$ & 196 & 0.29 \\
\hline
\end{tabular}




\begin{tabular}{|c|c|c|c|c|c|}
\hline 12. & Eugenol & 13.306 & $\mathrm{C}_{10} \mathrm{H}_{12} \mathrm{O}_{2}$ & 164 & 7.69 \\
\hline 13. & Geranyl acetate & 13.716 & $\mathrm{C}_{12} \mathrm{H}_{20} \mathrm{O}_{2}$ & 196 & 0.17 \\
\hline 14. & Methyleugenol & 14.182 & $\mathrm{C}_{11} \mathrm{H}_{14} \mathrm{O}_{2}$ & 178 & 0.30 \\
\hline 15. & $\begin{array}{l}\text { 1H-Cycloprop[e]azulene, } \\
\text { 1a,2,3,4,4a,5,6,7b-octahydro- } 1,1,4\end{array}$ & 14.379 & $\mathrm{C}_{15} \mathrm{H}_{24}$ & 204 & 0.16 \\
\hline 16. & Caryophyllene & 14.684 & $\mathrm{C}_{15} \mathrm{H}_{24}$ & 204 & 0.84 \\
\hline 17. & $\begin{array}{l}\text { PHENOL, } \\
\text { DIMETHYLETHYL)- }\end{array}$ & 16.255 & $\mathrm{C}_{14} \mathrm{H}_{22} \mathrm{O}$ & 206 & 0.17 \\
\hline 18. & Dodecanoic acid & 17.198 & $\mathrm{C}_{12} \mathrm{H}_{24} \mathrm{O}_{2}$ & 200 & 1.01 \\
\hline 19. & ETHYL PENTADECANOATE & 17.663 & $\mathrm{C}_{17} \mathrm{H}_{34} \mathrm{O}_{2}$ & 270 & 0.29 \\
\hline 20. & Tetradecanoic acid & 20.443 & $\mathrm{C}_{14} \mathrm{H}_{28} \mathrm{O}_{2}$ & 228 & 0.71 \\
\hline 21. & $\begin{array}{lll}\text { TETRADECANOIC } & \text { ACID, } & \text { ETHYL } \\
\text { ESTER } & & \\
\end{array}$ & 20.883 & $\mathrm{C}_{16} \mathrm{H}_{32} \mathrm{O}_{2}$ & 256 & 0.41 \\
\hline 22. & PENTADECANOIC ACID & 21.404 & $\mathrm{C}_{15} \mathrm{H}_{30} \mathrm{O}_{2}$ & 242 & 0.29 \\
\hline 23. & Neophytadiene & 21.556 & $\mathrm{C}_{20} \mathrm{H}_{38}$ & 278 & 0.96 \\
\hline 24. & 2-Pentadecanone, 6,10,14-trimethyl- & 21.628 & $\mathrm{C}_{18} \mathrm{H}_{36} \mathrm{O}$ & 268 & 0.56 \\
\hline 25. & ETHYL PENTADECANOATE & 21.825 & $\mathrm{C}_{17} \mathrm{H}_{34} \mathrm{O}_{2}$ & 270 & 0.23 \\
\hline 26. & Pentadecanoic acid & 21.945 & $\mathrm{C}_{15} \mathrm{H}_{30} \mathrm{O}_{2}$ & 242 & 0.69 \\
\hline 27. & 3,7,11,15-Tetramethyl-2-hexadecen-1-ol & 22.191 & $\mathrm{C}_{20} \mathrm{H}_{40} \mathrm{O}$ & 296 & 0.32 \\
\hline 28. & Pentadecanoic acid, ethyl ester & 22.370 & $\mathrm{C}_{17} \mathrm{H}_{34} \mathrm{O}_{2}$ & 270 & 0.38 \\
\hline 29. & Hexadecanoic acid, methyl ester & 22.837 & $\mathrm{C}_{17} \mathrm{H}_{34} \mathrm{O}_{2}$ & 270 & 0.22 \\
\hline 30. & 9-HEXADECENOIC ACID & 23.127 & $\mathrm{C}_{16} \mathrm{H}_{30} \mathrm{O}_{2}$ & 254 & 0.37 \\
\hline 31. & n-Hexadecanoic acid & 23.443 & $\mathrm{C}_{16} \mathrm{H}_{32} \mathrm{O}_{2}$ & 256 & 3.15 \\
\hline 32. & Ethyl 9-hexadecenoate & 23.492 & $\mathrm{C}_{18} \mathrm{H}_{34} \mathrm{O}_{2}$ & 282 & 0.38 \\
\hline 33. & $\begin{array}{l}\text { HEXADECANOIC ACID, ETHYL } \\
\text { ESTER }\end{array}$ & 23.797 & $\mathrm{C}_{18} \mathrm{H}_{36} \mathrm{O}_{2}$ & 284 & $\mathbf{7 . 5 0}$ \\
\hline 34. & (E)-9-Octadecenoic acid ethyl ester & 24.951 & $\mathrm{C}_{20} \mathrm{H}_{38} \mathrm{O}_{2}$ & 310 & 0.28 \\
\hline 35. & Heptadecanoic acid, ethyl ester & 25.152 & $\mathrm{C}_{19} \mathrm{H}_{38} \mathrm{O}_{2}$ & 298 & 0.33 \\
\hline 36. & 8,11,14-Eicosatrienoic acid, methyl ester & 25.239 & $\mathrm{C}_{21} \mathrm{H}_{36} \mathrm{O}_{2}$ & 320 & 0.22 \\
\hline 37. & $\begin{array}{ll}\text { 2-HEXADECEN-1-OL, } & 3,7,11,15- \\
\text { TETRAMETHYL-, [R-[R } & \end{array}$ & 25.398 & $\mathrm{C}_{20} \mathrm{H}_{40} \mathrm{O}$ & 296 & 0.59 \\
\hline 38. & 9,12-Octadecadienoyl chloride, (Z,Z)- & 25.849 & $\mathrm{C}_{18} \mathrm{H}_{31} \mathrm{C}_{\mathrm{l}} \mathrm{O}$ & 298 & 4.51 \\
\hline 39. & $\begin{array}{l}\text { trans,trans-9,12-Octadecadienoic acid, } \\
\text { propyl ester }\end{array}$ & 26.033 & $\mathrm{C}_{21} \mathrm{H}_{38} \mathrm{O}_{2}$ & 322 & 2.54 \\
\hline 40. & Dichloroacetic acid, tridec-2-ynyl ester & 26.115 & $\mathrm{C}_{15} \mathrm{H}_{24} \mathrm{C}_{12} \mathrm{O}_{2}$ & 306 & 6.70 \\
\hline 41. & (E)-9-Octadecenoic acid ethyl ester & 26.194 & $\mathrm{C}_{20} \mathrm{H}_{38} \mathrm{O}_{2}$ & 310 & 1.98 \\
\hline 42. & $\begin{array}{l}\text { OCTADECANOIC ACID, } \\
\text { ESTER }\end{array}$ & 26.455 & $\mathrm{C}_{20} \mathrm{H}_{40} \mathrm{O}_{2}$ & 312 & 1.50 \\
\hline 43. & $\begin{array}{l}\text { ETHYL }(9 Z, 12 Z)-9,12- \\
\text { OCTADECADIENOATE \# }\end{array}$ & 26.679 & $\mathrm{C}_{20} \mathrm{H}_{36} \mathrm{O}_{2}$ & 308 & 0.45 \\
\hline 44. & (R)-(-)-14-Methyl-8-hexadecyn-1-ol & 27.233 & $\mathrm{C}_{17} \mathrm{H}_{32} \mathrm{O}$ & 252 & 0.18 \\
\hline 45. & 9-OCTADECENAL, (Z)- & 28.028 & $\mathrm{C}_{18} \mathrm{H}_{34} \mathrm{O}$ & 266 & 0.22 \\
\hline 46. & $\begin{array}{l}\text { 1,5-PENT-2-ENE-3-METHYL-5-(2,6- } \\
\text { DIMETHYLHEPTH }\end{array}$ & 28.284 & $\mathrm{C}_{15} \mathrm{H}_{26} \mathrm{O}_{2}$ & 238 & 0.82 \\
\hline 47. & 4,8,12,16-Tetramethylheptadecan-4-olide & 28.395 & $\mathrm{C}_{21} \mathrm{H}_{40} \mathrm{O}_{2}$ & 324 & 0.35 \\
\hline 48. & (E)-9-Octadecenoic acid ethyl ester & 28.582 & $\mathrm{C}_{20} \mathrm{H}_{38} \mathrm{O}_{2}$ & 310 & 1.67 \\
\hline 49. & $\begin{array}{lll}\text { HEPTADECANOIC } & \text { ACID, } & \text { ETHYL } \\
\text { ESTER } & & \\
\end{array}$ & 28.866 & $\mathrm{C}_{19} \mathrm{H}_{38} \mathrm{O}_{2}$ & 298 & 0.70 \\
\hline 50. & $\begin{array}{l}\text { 1,3-Cyclopentadiene, } \\
\text { (dimethylamino)propyl]- }\end{array}$ & 29.457 & $\mathrm{C}_{10} \mathrm{H}_{17} \mathrm{~N}$ & 151 & 0.10 \\
\hline 51. & 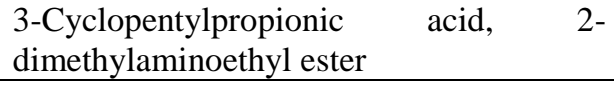 & 29.520 & $\mathrm{C}_{12} \mathrm{H}_{23} \mathrm{NO}_{2}$ & 213 & 0.29 \\
\hline 52. & 1,2-15,16-Diepoxyhexadecane & 29.669 & $\mathrm{C}_{16} \mathrm{H}_{30} \mathrm{O}_{2}$ & 254 & 0.48 \\
\hline 53. & Behenic alcohol & 29.844 & $\mathrm{C}_{22} \mathrm{H}_{46} \mathrm{O}$ & 326 & 1.36 \\
\hline
\end{tabular}




\begin{tabular}{|c|c|c|c|c|c|}
\hline 54. & 1,2-BENZENEDICARBOXYLIC ACID & 30.171 & $\mathrm{C}_{24} \mathrm{H}_{38} \mathrm{O}_{4}$ & 390 & 0.25 \\
\hline 55. & (E)-9-Octadecenoic acid ethyl ester & 30.465 & $\mathrm{C}_{20} \mathrm{H}_{38} \mathrm{O}_{2}$ & 310 & 0.68 \\
\hline 56. & Docosanoic acid, ethyl ester & 30.671 & $\mathrm{C}_{24} \mathrm{H}_{48} \mathrm{O}_{2}$ & 368 & 0.51 \\
\hline 57. & 1-Cyclohexyldimethylsilyloxybutane & 30.728 & $\mathrm{C}_{12} \mathrm{H}_{26} \mathrm{OSi}$ & 214 & 0.48 \\
\hline 58. & $\begin{array}{l}\text { Fumaric acid, 2-dimethylaminoethyl } \\
\text { octadecyl ester }\end{array}$ & 31.270 & $\mathrm{C}_{26} \mathrm{H}_{49} \mathrm{NO}_{4}$ & 439 & 0.09 \\
\hline 59. & Hexacosylheptafluorobutyrate & 31.613 & $\mathrm{C}_{30} \mathrm{H}_{53} \mathrm{~F}_{7} \mathrm{O}_{2}$ & 578 & 1.57 \\
\hline 60. & (E)-9-Octadecenoic acid ethyl ester & 32.317 & $\mathrm{C}_{20} \mathrm{H}_{38} \mathrm{O}_{2}$ & 310 & 0.32 \\
\hline 61. & Ethyl tetracosanoate & 32.564 & $\mathrm{C}_{26} \mathrm{H}_{52} \mathrm{O}_{2}$ & 396 & 1.52 \\
\hline 62. & 1-Cyclohexyldimethylsilyloxybutane & 32.645 & $\mathrm{C}_{12} \mathrm{H}_{26} \mathrm{OSi}$ & 214 & 1.25 \\
\hline 63. & Squalene & 32.784 & $\mathrm{C}_{30} \mathrm{H}_{50}$ & 410 & 0.20 \\
\hline 64. & Benzenepropanoic acid, octadecyl ester & 33.392 & $\mathrm{C}_{27} \mathrm{H}_{46} \mathrm{O}_{2}$ & 402 & 0.33 \\
\hline 65. & 1-Heptacosanol & 33.844 & $\mathrm{C}_{27} \mathrm{H}_{56} \mathrm{O}$ & 396 & 1.18 \\
\hline 66. & $\begin{array}{lll}\text { OCTADECANOIC } & \text { ACID, } & \text { ETHYL } \\
\text { ESTER } & & \end{array}$ & 35.146 & $\mathrm{C}_{20} \mathrm{H}_{40} \mathrm{O}_{2}$ & 312 & 0.88 \\
\hline 67. & $\begin{array}{l}\text { Stigmasta-5,22-dien-3-ol, } \\
\text { (3.beta.)- }\end{array}$ & 36.558 & $\mathrm{C}_{31} \mathrm{H}_{50} \mathrm{O}_{2}$ & 454 & 0.53 \\
\hline 68. & Stigmast-5-en-3-ol, oleate & 36.936 & $\mathrm{C}_{47} \mathrm{H}_{82} \mathrm{O}_{2}$ & 678 & 0.29 \\
\hline 69. & 1-Heptacosanol & 37.075 & $\mathrm{C}_{27} \mathrm{H}_{56} \mathrm{O}$ & 396 & 0.65 \\
\hline 70. & Cholesterol & 37.474 & $\mathrm{C}_{27} \mathrm{H}_{46} \mathrm{O}$ & 386 & 1.25 \\
\hline 71. & ERGOST-5-EN-3-OL, (3.BETA.,24R)- & 39.753 & $\mathrm{C}_{28} \mathrm{H}_{48} \mathrm{O}$ & 400 & 2.38 \\
\hline 72. & Stigmasterol & 40.394 & $\mathrm{C}_{29} \mathrm{H}_{48} \mathrm{O}$ & 412 & 2.86 \\
\hline 73. & STIGMAST-5-EN-3-OL, (3.BETA.)- & 42.018 & $\mathrm{C}_{29} \mathrm{H}_{50} \mathrm{O}$ & 414 & 7.14 \\
\hline 74. & 4-Campestene-3-one & 43.145 & $\mathrm{C}_{28} \mathrm{H}_{46} \mathrm{O}$ & 398 & 1.90 \\
\hline 75. & 4,22-Stigmastadiene-3-one & 43.920 & $\mathrm{C}_{29} \mathrm{H}_{46} \mathrm{O}$ & 410 & 1.40 \\
\hline 76. & .gamma.-Sitostenone & 46.035 & $\mathrm{C}_{29} \mathrm{H}_{48} \mathrm{O}$ & 412 & 4.10 \\
\hline 77. & $\begin{array}{l}\text { Cholestan-3-one, 4,4-dimethyl-, } \\
\text { (5.alpha.)- }\end{array}$ & 48.559 & $\mathrm{C}_{29} \mathrm{H}_{50} \mathrm{O}$ & 414 & 3.58 \\
\hline 78. & $\begin{array}{l}\text { CYCLODODECANONE, } \\
\text { HYDROXYBUTYL)-2-NIT }\end{array}$ & 49.648 & $\mathrm{C}_{16} \mathrm{H}_{29} \mathrm{NO}_{4}$ & 299 & 2.35 \\
\hline 79. & Stigmastane-3,6-dione, (5.alpha.)- & $\mathbf{5 2 . 5 5 9}$ & $\mathrm{C}_{29} \mathrm{H}_{48} \mathrm{O}_{2}$ & 428 & $\mathbf{5 . 0 5}$ \\
\hline
\end{tabular}

Figure :2.6. Structure Of Compounds Isolated In Lepidium sativum Whole Plant Extract

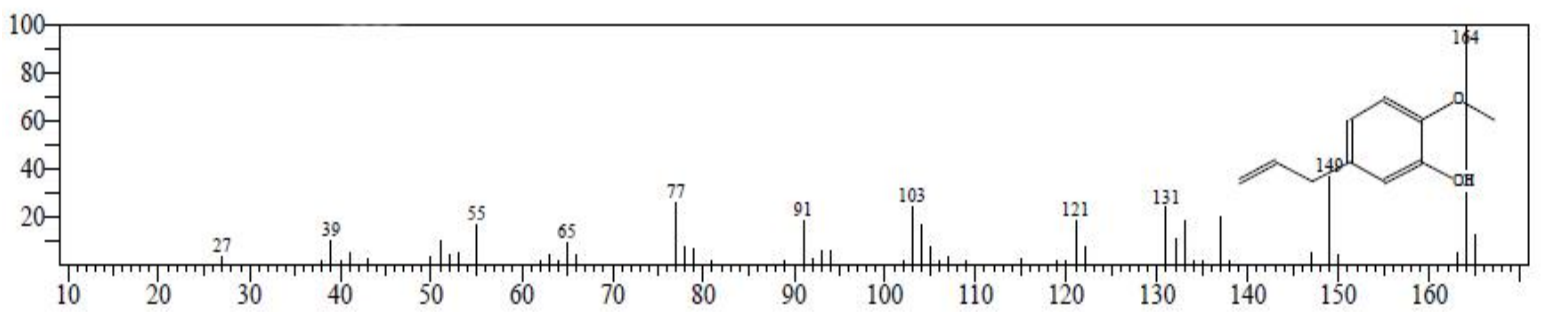

Fig 2.6.1:- Mass Spectrum of Eugenol

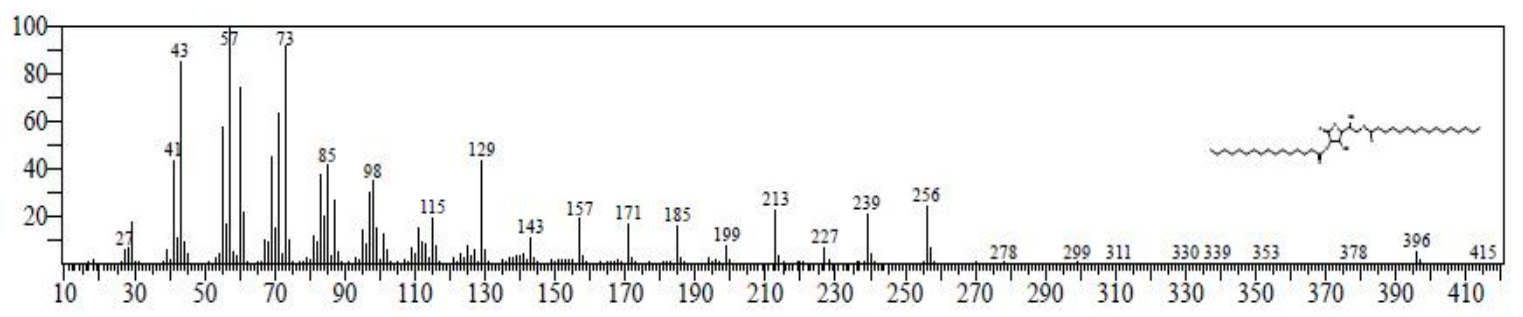

Fig 2.6.2.:- Mass Spectrum of n-Hexadecanoic acid 


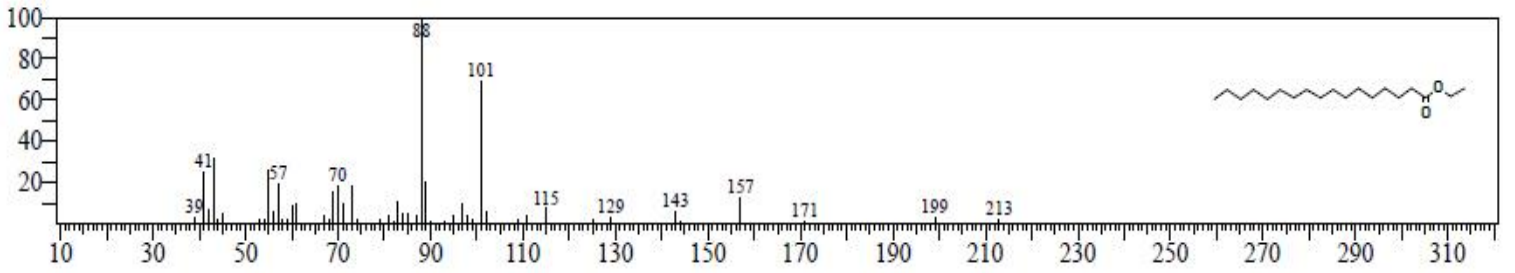

Fig 2.6.3:- Mass Spectrum of Hexadecanoic Acid, Ethyl Ester

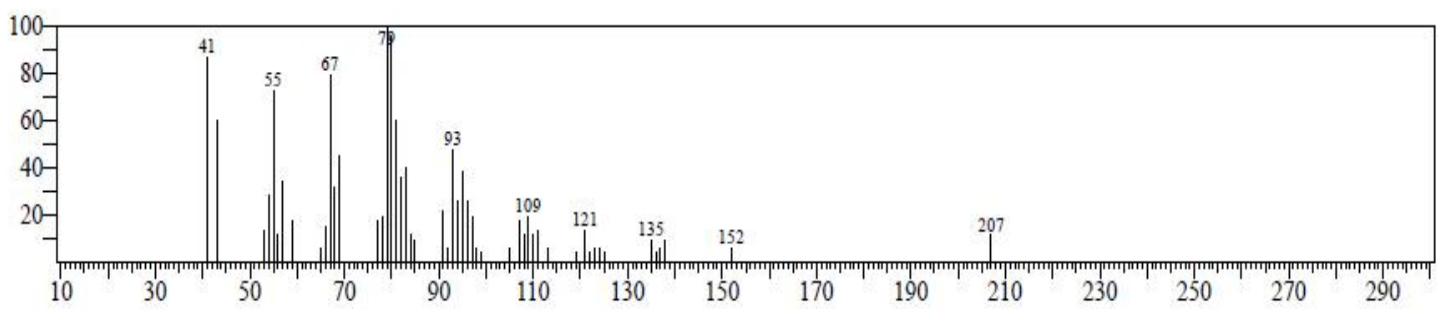

Fig 2.6.4:- Mass Spectrum of 9,12-Octadecadienoyl chloride, (Z,Z)-

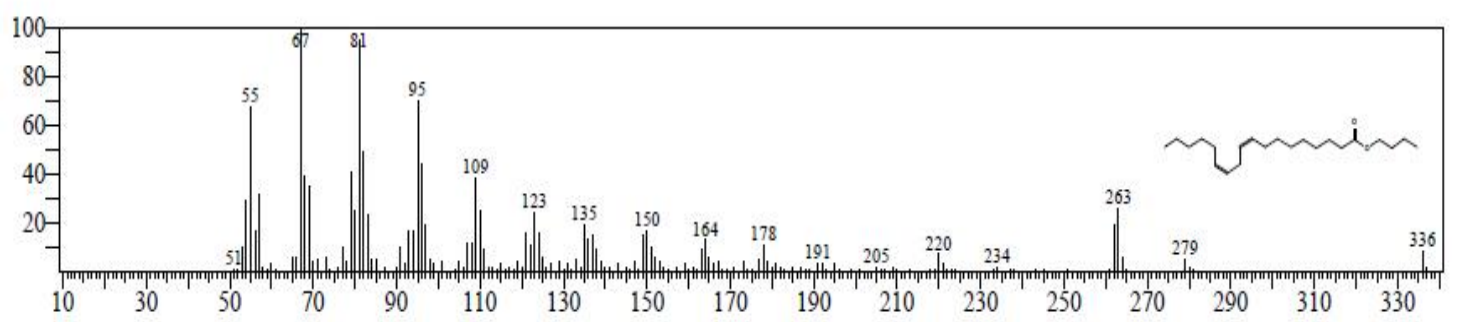

Fig 2.6.5 :- Mass Spectrum of trans,trans-9,12-Octadecadienoic acid, propyl ester

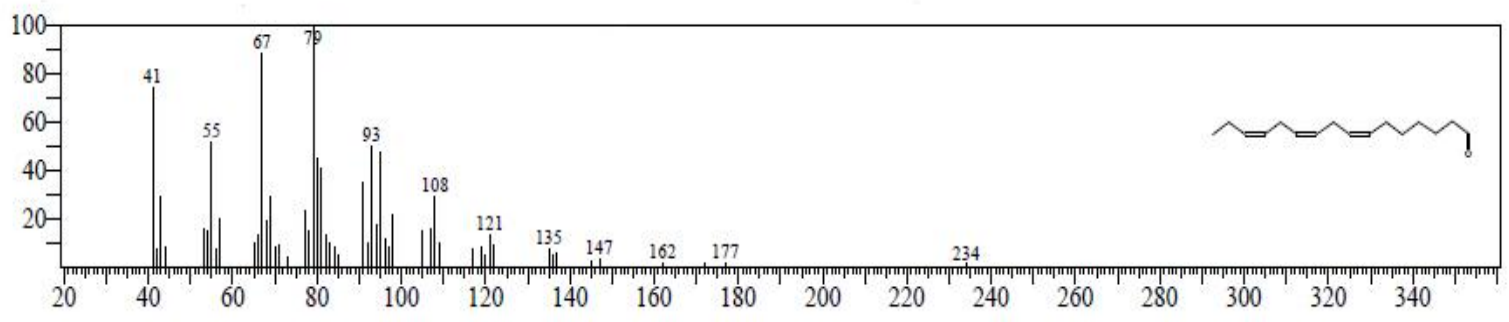

Fig 2.6.6:- Mass Spectrum of Dichloroacetic acid, tridec-2-ynyl ester

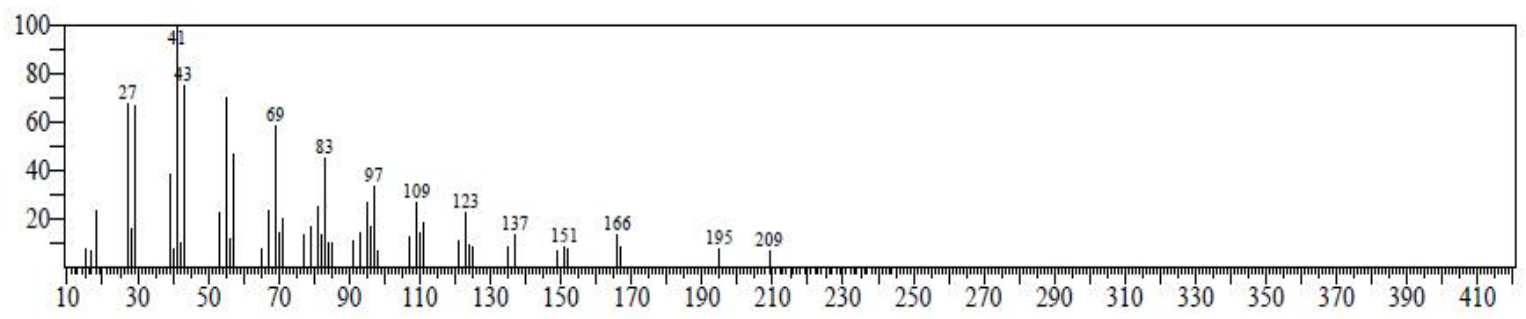

Fig 2.6.7:- Mass Spectrum of ERGOST-5-EN-3-OL, (3.BETA.,24R)- 


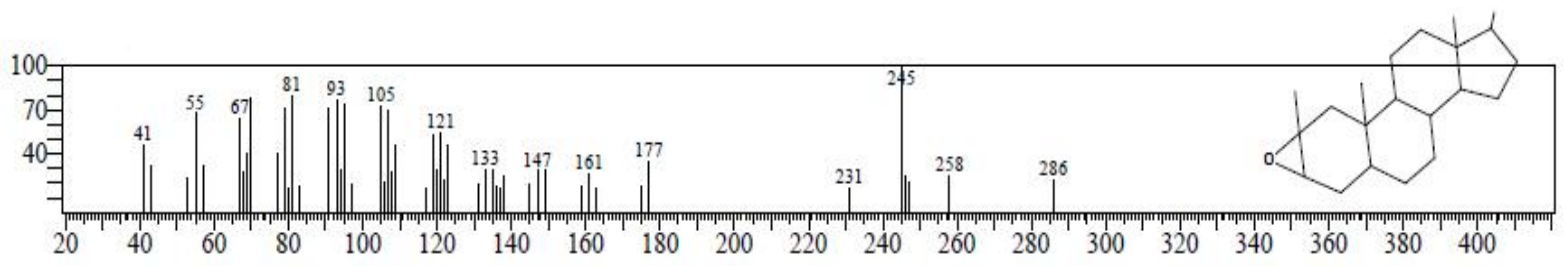

Fig 2.6.8:- Mass Spectrum of Stigmasterol

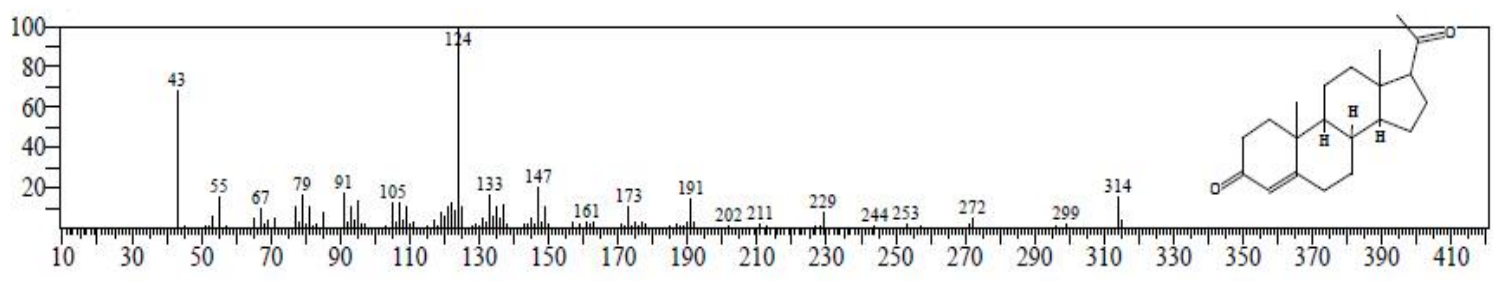

Fig 2.6.9:- Mass Spectrum of Stigmast-5-EN-3-OL, (3.BETA.)-

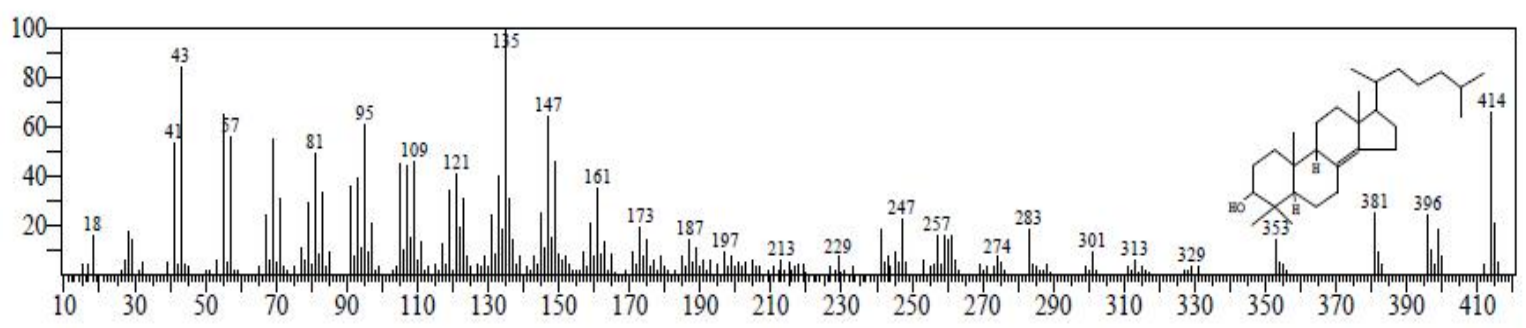

Fig 2.6.10:- Mass Spectrum of.gamma.-Sitostenone

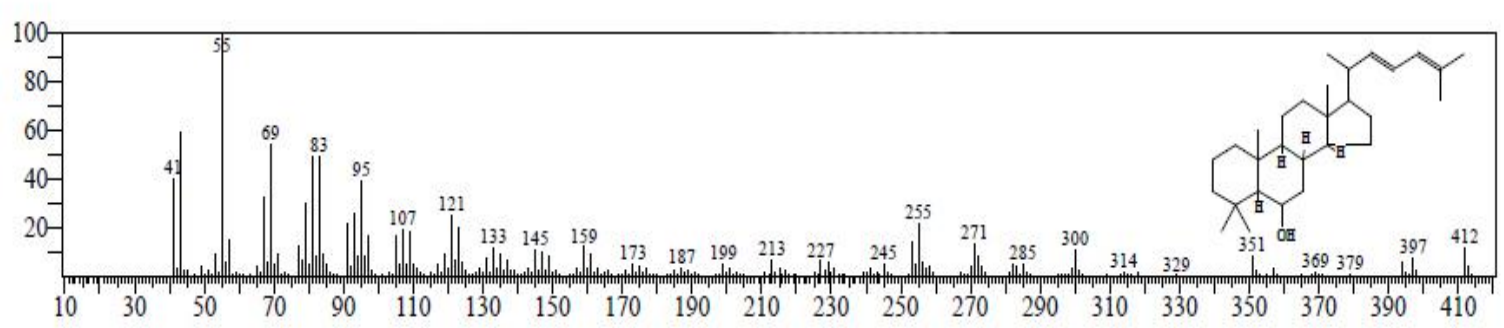

Fig 2.6.11:- Mass Spectrum of Cholestan-3-one, 4,4-dimethyl-, (5.alpha.)-

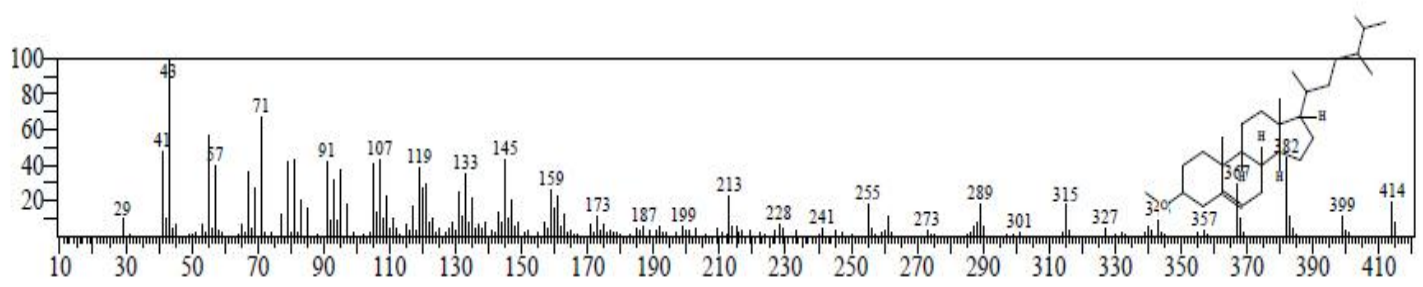

Fig 2.6.12 :- Mass Spectrum of Cyclododecanone, 2-(3-Hydroxybutyl)-2-NIT 


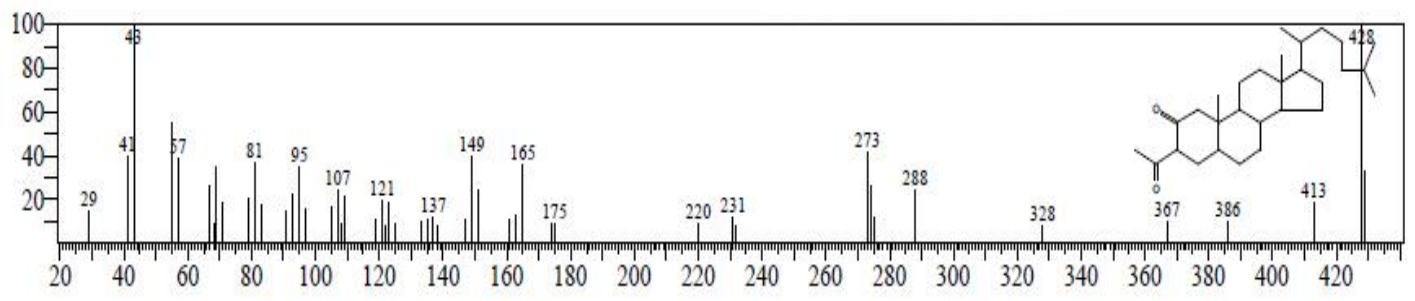

Fig 2.6.12 :- Mass Spectrum of Stigmastane-3,6-dione, (5.alpha.)-

\section{References:-}

1. Cerutti, PA 1991, 'Oxidant stress and carcinogenesis’ European Journal of Clinical Investigation, vol. 21, no. 1, pp. 1-5.

2. Shukla, AK, Bigoniya, P \&Soni, P 2015, 'Hypolipidemic Activity of LepidiumSativumLinn. Seed in Rats',Journal of Pharmacy and Biological Sciences, vol.10, pp. 13-22.

3. Cassidy, Frederic Gomes, Hall \& Joan Houston 2002, 'Dictionary of American regional English', Harvard University Press, pp. 97.

4. Radwan, HM, El-Missiry, MM, Al-Said, WM, Ismail, AS, Abdel-Shafeek, KA \&Seif-El-Nasr, MM 2007, Research Journal of Medicine and Medicinal Sciences, vol. 2, pp. 127- 132.

5. Martins, AP, Salgueiro, L \&Goncalves, MJ 2001, 'Essential oil composition and antimicrobial activity of three Zingiberaceae from S. Tome e Principle', PlantaMedica, vol. 67, pp. 580-584.

6. AH, N \&Aysel, U. 2003, 'Investigation of the Antimicrobial Activity of Some Streptomyces Isolates', Turkish Journal of Biology, vol. 27, pp. 79-84.

7. Solomon GirmayBerehe\&AmanDekeboBoru 2014, 'Phytochemical Screening and Antimicrobial activities af Crude Extract of LepidiumSativiumSeeds Grown in Ethiopia', International Journal of Pharmaceutical Sciences and Research, vol. 5, no.10, pp. 4182-4187.

8. RizwanAhamad, MohdMujeeb, Firoz Anwar \&Aftab Ahmad 2015, 'Phytochemical analysis and evaluation of anti-oxidant activity of methanolic extract of LepidiumsativumL. seeds', Der Pharmacia Lettre, vol. 7, no. 7, pp. 427-434.

9. Muhammad Rizwan-ul-Haq, Mei Ying Hu, Muhammad Afzal\& Muhammad Hamid 2012, Journal of Natural Products, vol. 52, no. 2, pp.325-31.

10. Akash, MS, Rehman, K, Tariq, M, Chen, S 2017, ' Zingiberofficinaleand type 2 diabetes mellitus: Evidence from experimental studies', Critical Reviews in Eukaryotic Gene Expression, vol. 25, no. 2.

11. Kassie, F, Rabot, S, Uhl, M, Huber,W, Qin, HM, Helma, C, Hermann,RS \&Knasmuller , S 2002, 'Chemoprotective effects of gardencress (Lepidiumsativum) and its constituents towards2-amino-3-methylimidazo[4, 5-f]quinoline (IQ)-induced genotoxiceffects and colonic preneoplastic lesions',Carcinogenesis, vol. 23, pp. 1155-1161.

12. Hussein, HM, Hameed, IH \&Ibraheem, OA 2017, 'Antimicrobial Activity and spectral chemical analysis of methanolic leaves extract of AdiantumCapillusVeneris using GC-MS and FT-IR spectroscopy’, International Journal of Pharmacognosy and Phytochemical Research, vol. 8, no. 3, pp. 369-385.

13. KshitijChauhan, Nishteswar, K \&Chauhan, MG 2012, 'Pharmacognostical Evaluation of Seeds of LepidiumsativumLinn',International Journal of Pharmaceutical \& Biological Archives,vol. 3, no.3, pp. 627-631.

14. Yadav, YC, Srivastava, DN, Saini,V, Seth, AK, Ghelani, TK, Malik, A\& Kumar S 2011, 'In vitro antioxidant activities of ethanolic extract of Lepidiumsativum L. seeds', International Journal of Pharma Sciences, vol. 1, pp. 965-974.

15. Prajapati, VD, Maheriya, PM, Jani, GK, Patil, PD \& Patel, BN 2014, 'Lepidiumsativum

16. Linn.: a current addition to the family of mucilage and its applications', International

17. Journal of Biological Macromolecules, vol. 65, pp. 72-80. 